\title{
Die Krise Kaliforniens: Ende in Sicht? Der Beitrag der direkten Demokratie zur Krisenüberwindung
}

\author{
Hermann K. Heußner
}

\section{A. Einleitung}

In der deutschen Diskussion über direkte Demokratie spielt Kalifornien wegen seiner intensiv praktizierten Volksgesetzgebung eine herausgehobene Rolle. ${ }^{1}$ Es wunderte daher nicht, dass die Krise des politischen Systems, die dort seit Jahrzehnten zu beobachten war und sich u.a. in wiederkehrenden, massiven Budgetproblemen spiegelte, häufig der dortigen Volksgesetzgebung angelastet wurde. Bei näherem Hinsehen stellt sich heraus, dass nicht Volksgesetzgebung an sich dafür verantwortlich ist, sondern einerseits die amerikanische und insbesondere kalifornische Ausprägung dieses Prinzips, die sich stark vom schweizerischen und deutschen System unterscheidet, und anderseits schwerwiegendes Parlamentsversagen im Zusammenspiel mit der starken Polarisierung der kalifornischen Parteien. ${ }^{2}$

Nunmehr verzeichnen in Kalifornien seit 2008 Abstimmungsvorlagen in Volksentscheiden Siege - insgesamt sieben -, die einschneidende Änderungen des politischen Systems versprechen. ${ }^{3}$ Leiten sie das Ende der kalifornischen Krise ein? Leistet gerade direkte Demokratie einen Beitrag dazu, die Probleme des politischen Systems Kaliforniens zu lösen? Dem soll im Folgenden nachgegangen werden. Zunächst werden die Vorlagen behandelt, welche die Arbeitsfähigkeit des Parlaments verbessern sollen (B), dann diejenigen, welche die Staatseinnahmen erhöhen und die Staatsausgaben ver-

1 Zur Bedeutung Kaliforniens s. H.K. Heußner, Die Krise Kaliforniens - Die Schuld der direkten Demokratie?, in: L.P. Feld u.a. (Hrsg.), Jahrbuch für direkte Demokratie 2011, 2012, S. 175-233 (175 f., 179 f.).

2 Ausführlich dazu Heußner, Krise (Fn. 1), S. 175 ff.

3 Dies sind: 2008 Proposition 11 (Wahlkreiseinteilung, VI); Prop. 28, Amtszeiten für Abgeordnete, VI); 2010 Prop. 14 (Vorwahlen, VR); Prop. 25 (Mehrheitserfordernis für Budgetbeschluss, VI); 2012 Prop. 30 (Steuern, VI); Prop. 39 (Steuern, GI); Prop. 36 (Strafrecht, GI). VI: Verfassungsinitiative, VR: Verfassungsreferendum; GI: Gesetzesinitiative. 
mindern (C). Abschließend geht es um die Wirksamkeit der Gesetzesänderungen und ein Fazit für Deutschland (D).

\section{B. Arbeitsfähigkeit des Parlaments}

\section{Kompromissfähigkeit}

Eine wesentliche Ursache der kalifornischen Krise besteht darin, dass das Parlament zu keinen Kompromissen fähig ist. Dies liegt nicht daran, dass sich die beiden Parlamentskammern aufgrund unterschiedlicher Parteimehrheiten blockierten. Denn in Kalifornien sind beide Parlamentskammern seit Jahrzehnten fest in demokratischer Hand. Lediglich von 1994 bis 1996 hatte die republikanische Partei kurzzeitig eine Mehrheit in der unteren Kammer, der State Assembly. ${ }^{4}$ Das Problem besteht vielmehr darin, dass sich die demokratische Parlamentsmehrheit mit der jeweiligen republikanischen Parlamentsminderheit häufig nicht auf Budget- und Abgabenkompromisse einigen konnte. Dies ist in Kalifornien aber unumgänglich, weil dort sowohl Steuererhöhungen als auch die jährliche Verabschiedung des Staatshaushalts jeweils eine Zweidrittelmehrheit benötig(t)en. Die Parlamentsminderheit besitzt bzw. besaß also Vetomacht, sofern sie nur mindestens ein Drittel der Sitze plus einen Sitz in mindestens einer Parlamentskammer hält. Zwar sind in einem präsidentiellen Zweikammersystem Blockaden (gridlock) an sich nichts Ungewöhnliches. Ungewöhnlich ist jedoch die Vetoposition einer Drittelminderheit. Blockaden zu überwinden, ist umso schwieriger, je polarisierter die Parteien sind. Und ausgerechnet in Kalifornien sind Republikaner und Demokraten im Parlament ideologisch mit großem Abstand weiter auseinander als in jedem anderen US-Gliedstaat. ${ }^{5}$ In Kalifornien ist das Parlament für Blockaden also ganz besonders anfällig.

4 R. Brownstein, How the democrats are taking over California, in: National Journal, 18.1.2013, http:/www.nationaljournal.com/thenextamerica/politics/how-the-democrats-are-taking-over-california-20130118?print=true (16.4.2013); E. Gould, Is California's Supermajority an Omen for the Rest of the U.S.?, in: Time Magazine, 20.11.2012, http://nation.time.com/2012/11/20/is-californias-democratic-supermajority-an-omen-for-the-rest-of-the-u-s/ (22.3.2013). Zur genauen Sitzverteilung in den beiden Parlamentskammern s. Jahrbuch des Council of State Government (Hrsg.), The Book of the States, jeweils Tabelle 3.3.

5 E. McGee/S. Masket/B. Shor/S. Rogers/N. McCarty, A Primary Cause of Partisanship? Nomination Systems and Legislator Ideology, in: American Journal of Political Science 58 (2014), S. 337 ff., Fig. 1. http://www.princeton.edu/ nmccarty/ 
Ein Reformansatz besteht deshalb darin, größere parlamentarische Kompromissbereitschaft zu erzeugen. Dafür sind auf dem Weg der Volksgesetzgebung bzw. direkten Demokratie zwei Wahlrechtsänderungen beschlossen worden: die Reform des Verfahrens der Wahlkreisneueinteilung und die Änderung des Vorwahlsystems.

\section{Wahlkreisneueinteilung}

In den USA müssen die Wahlkreise für die Parlamente mindestens alle zehn Jahre neu eingeteilt werden, um den jeweils eingetretenen Bevölkerungsänderungen gerecht zu werden. ${ }^{6}$ Dabei haben die Staaten relativ großen Spielraum. $^{7}$ Da der Zuschnitt der Wahlkreise für die Wahl- bzw. Wiederwahlchancen der Kandidaten entscheidend sein kann, ist insbesondere von Bedeutung, wer die Neueinteilungen vornimmt. In der Dekade ab 2000 war dies in den meisten Staaten das Landesparlament selbst, während in einer Minderheit von Staaten Kommissionen unterschiedlicher Art diese Aufgabe zum Teil oder allein inne hatten. ${ }^{8}$ Auch in Kalifornien hatte das Parlament nach der Volkszählung des Jahres 2000 die Wahlkreise 2001 neu eingeteilt. Dies führte für beide Parteien zu ,sicheren“ Wahlkreisen und schützte so die Mandatsinhaber. ${ }^{9}$ In allen folgenden Wahlen für das kalifornische Parlament kam es deshalb lediglich in sieben von insgesamt 612 Fällen vor, dass eine Partei einen Wahlkreis verlor. ${ }^{10}$

PrimarySystems.pdf (16.4.2013). Die Polarisierung des US-Kongresses ist demgegenüber wesentlich weniger ausgeprägt, ebda.

6 Näher T. Donovan/C.Z. Mooney/D.A. Smith, State and Local Politics. Institutions and Reform, Belmont 2009, S. 202 ff.

7 Dies., ebda., S. 204 f.

8 M. Cullen/M. Davis, 5 Trends Shaping Redistricting, in: State Legislatures, 1011/2012, S. 32; E.A. Lindgren, Commissions and Competition in the 2002 through 2010 US House Elections, S. 4 f., http://2012sppconference.blogs.rice.edu/files/ 2012/02/Com-missions-and-Competition-in-the-2002-through-2010-US-House-

Elections.docx (16.4.2013); vgl. auch Council of State Governments, State Reditricting Decisions, 2011, http://www.csg.org/pubs/capitolideas/enews/issue59/State_ Redistricting_Chart.pdf (16.4.2013).

9 K. DeBow/J.C. Syer, Power and Politics in California, 9. Aufl. New York 2009, S. 58; L.L. Giventer, Governing California, 2. Aufl. Boston u.a. 2009, S. 25 f.

10 B. Ambühl, Die Kunst, ,gerechte“ Wahlkreise zu schaffen, in: NZZ, Intern. Ausg. v. 4.8. 2011, S. 5. 
Gegen diese Einzementierung sicherer Wahlkreise richtete sich im Jahr 2008 das siegreiche Volksbegehren Proposition 11. ${ }^{11}$ Es führt eine 14köpfige Bürgerkommission ein, die anstelle des Parlaments für die Wahlkreisneueinteilung zuständig ist (Citizens Redistricting Commission). ${ }^{12}$ Die Initiatoren gehen davon aus, dass Abgeordnete, die keiner Konkurrenz ausgesetzt sind und sich daher nicht um Wählerstimmen bemühen müssen, den Wählern nicht mehr verantwortlich sind. Die Abgeordneten arbeiteten deshalb im Parlament auch nicht problemlösungsorientiert zusammen, sondern dienten eher Sonderinteressen. ${ }^{13}$ Die Bürgerkommission ist verpflichtet, die Wahlkreise u.a. so einzuteilen, dass deren Zuschnitt weder begünstigend noch diskriminierend für Amtsinhaber oder sonstige Kandidaten oder bestimmte Parteien wirkt. ${ }^{14}$ Das neue Verfahren ist mittlerweile in der Bevölkerung fest verankert. Proposition 27, welche die Reform wieder rückgängig machen wollte, scheiterte 2010 mit 59,5\% Nein-Stimmen. ${ }^{15}$ Der Versuch der Republikaner 2012, die Wahlkreiseinteilung im Wege des neuen Verfahrens für die 40 Wahlkreise des State Senate durch ein fakultatives Referendum zu stoppen, misslang ebenfalls (Proposition 40). Die Neueinteilung bekam 72\% Zustimmung. ${ }^{16}$

11 Zu Proposition 11 s. D. Bowen, California Secretary of State (Hrsg.), Voter Information Guide, General Election, 4.11.2008, S. 70 ff., 137 ff., http://librarysource. uchastings.edu/ballot_pdf/2008g.pdf (10.4.2013).

12 Vgl. auch Homepage der Kommission, http://wedrawthelines.ca.gov/ (20.4.2003).

13 Argument in favor of proposition 11, in: Bowen, Guide 2008 (Fn. 11), S. 72 f. und Sec. 1 (Findings and Purpose) des vorgeschlagenen „Voters First Act“, ebda., S. 137.

14 Analyse des neutralen Legislative Analyst, in: Bowen, Guide 2008 (Fn. 11), S. 70 f.; Art. XXI Sec. 2 (d), (e) California Constitution. - Zur Zusammensetzung und Arbeitsweise der unabhängigen Bürgerkommission s. näher Sections 8252, 8353 (a) (7) California Government Code; Art. XXI Sec. 2 (c) (5) California Constitution. Gegen die Wahlkreisneueinteilungen der Kommission kann das fakultative Referendum ergriffen werden, Art. XXI Sec. 2 (i) California Constitution. - Zum Abstimmungskampf vgl. H.K. Heußner, Direkte Demokratie in den US-Gliedstaaten im Jahr 2008, in: L.P. Feld u.a. (Hrsg.), JdD 2009, 2010, S. 165-204 (180).

15 Näher H.K. Heußner, Direkte Demokratie in den US-Gliedstaaten in den Jahren 2010 und 2011, in: L.P. Feld u.a. (Hrsg.), JdD 2012, 2013, S. 181-228 (198 f.).

16 California Secretary of State, Statement of Vote, November 6, General Election, S. 72, http://www.sos.ca.gov/elections/sov/2012-general/sov-complete.pdf (23.6.2014). 


\section{Vorwahlen}

Der zweite „Hebel“, um zu kompromissbereiteren Abgeordneten zu kommen, setzt bei den Vorwahlen an. 2009 beschloss das Parlament auf Betreiben des moderaten republikanischen State Senator Abel Maldonado, das „Top-Two Open Primary“ System mittels Verfassungsänderung einzuführen. ${ }^{17}$ In dem neuen Vorwahlsystem erscheinen alle Kandidaten auf einem einzigen Stimmzettel. „Alle Wähler haben die Möglichkeit, (irgend)einen Kandidaten irgendeiner Partei zu wählen. Nur die Kandidaten mit der höchsten und zweithöchsten Stimmenzahl erscheinen dann auf dem Wahlzettel der Hauptwahlen. Diese können derselben Partei angehören. Auf diese Weise können auch moderatere Kandidaten, die unter den Anhängern der eigenen Partei weniger Chancen haben, in die Hauptwahl gelangen, wo sie ggf. auch von Anhängern der anderen Partei unterstützt werden. Die so gewählten, eher moderaten Abgeordneten könnten dann eher gewillt sein, im Parlament nach Kompromissen zu suchen. “18 Das Volk stimmte der Vorlage 2010 mit $53,8 \%$ Ja-Stimmen zu. ${ }^{19}$

\section{Zweidrittelmehrheitserfordernis für den Haushalt}

Wie dargestellt, musste bisher in Kalifornien das Parlament dem Haushalt mit einer Zweidrittelmehrheit zustimmen. ${ }^{20}$ Dies trug wesentlich dazu bei, dass der Staatshaushalt zwischen 1980 und 2010 lediglich zehnmal rechtzeitig verabschiedet wurde und Kalifornien 1992 und 2009 zeitweilig zahlungsunfähig war. ${ }^{21} 2010$ schafften die Wähler diese Hürde ab. Die Verfassungsinitiative Proposition 25 war mit 55,1\% Ja-Stimmen erfolgreich. Seitdem ist nur eine einfache Mehrheit erforderlich. ${ }^{22}$

Die von Proposition 131978 eingeführte Zweidrittel-Supermajority für parlamentarische Steuererhöhungen ist bisher jedoch unangetastet geblieben. Ganz im Gegenteil konnte sich 2010 die Verfassungsinitiative Proposition

\section{Heußner, Krise (Fn. 1), S. 215 f.}

18 Ebda., S. 216.

19 Ebda., S. 215.

20 California Constitution Art. IV Sect. 12 (d) a.F.

21 Heußner, Krise (Fn. 1), S. 211.

22 Näher Heußner, US-Gliedstaaten 2010-2011 (Fn. 15), S. 192 f. 
26 mit 52,5\% Ja-Stimmen durchsetzen, die nunmehr auch für bestimmte Gebühren und Beiträge das Zweidrittelmehrheitserfordernis einführte. ${ }^{23}$

\section{Expertise und Verantwortungsbereitschaft der Abgeordneten}

Die Frustration der Bürger über die Dysfunktionalität der kalifornischen Politik und über ihre Abgeordneten, welche die Probleme nicht oder nur ungenügend lösten, ${ }^{24}$ führte 1990 zur Verabschiedung von „Term Limits“. Die Verfassungsinitiative Proposition 140 gewann mit 52,2\% Ja-Stimmen. Sie begrenzte die Anzahl der möglichen Amtsperioden der Abgeordneten. Diese waren mit insgesamt lediglich acht (Senate) bzw. sechs Jahren (Assembly) sehr kurz. Die Expertise der Abgeordneten sank daraufhin. Die Anreize, eine auf längere Sicht verantwortungsvolle Politik zu betreiben, gingen zurück. ${ }^{25}$

2012 war die Verfassungsinitiative Proposition 28 mit 61\% Ja-Stimmen erfolgreich. Nunmehr ist es zulässig, insgesamt zwölf Jahre Abgeordneter zu sein. Wie viele Jahre davon im Abgeordnetenhaus oder im Senat zugebracht werden, ist irrelevant. ${ }^{26}$ Dies bedeutet, dass die Abgeordneten wesentlich länger Zeit haben, Expertise aufzubauen. Gleichzeitig müssen sie damit rechnen, auch als Abgeordnete für die Folgen ihrer Politik von den Wählern zur Verantwortung gezogen zu werden. ${ }^{27}$

\section{C. $\quad$ Staatseinnahmen und-ausgaben}

Die Blockaden des Parlaments und die Reaktion des Volkes haben in der Vergangenheit dazu geführt, dass die Staatseinnahmen zu niedrig waren und die Staatsausgaben z.T. Fehlallokationen darstellten. ${ }^{28}$

23 Ebda., S. $189 \mathrm{f}$.

24 Vgl. auch H.K. Heußner, Demokratiereformen durch das Volk, in: RuP 36 (2000), S. 149-155 (151).

25 Heußner, Krise (Fn. 1), S. 226 f.

26 D. Bowen, California Secretary of State, California General Election, Tuesday, November 6, 2012, Official Voter Information Guide, S. 8, 24. http://vig.cdn.sos.ca. gov/ 2012/ general/pdf/complete-vig-v2.pdf (10.4.2013).

27 Vgl. Bowen, Guide 2012 (Fn. 26), Argument in favor of proposition 28, S. 10.

28 Näher Heußner, Krise (Fn. 1), S. 189 ff., 203 ff. 


\section{Steuererhöhungen}

\section{Erhöhung der Einkommen- und der Verkaufsteuer}

Ein gravierendes Problem der kalifornischen Krise liegt in den Hürden, die Einnahmen des Staates zu erhöhen. Denn für Steuererhöhungen ist im Parlament wiederum eine Zweidrittelmehrheit erforderlich. ${ }^{29}$ Um diese Hürde zu umgehen, ist es notwendig, mit Hilfe von Volksbegehren das Volk selbst entscheiden zu lassen. Dies geschah 2012 in Form der Verfassungsinitiative Proposition $30 .{ }^{30}$ Sie sieht vor, dass die Verkaufsteuer für vier Jahre um 0,25 Prozentpunkte angehoben wird. Bis zur Erhöhung betrug die durchschnittliche Verkaufsteuer in Kalifornien - staatliche und kommunale zusammen gerechnet - ca. 8\%. ${ }^{31}$ Die kalifornische Einkommensteuer steigt für sieben Jahre für Einkommen ab 250.000 Dollar von 9,3\% auf 10,3\%, ab 300.000 Dollar auf $11,3 \%$ und über 500.000 Dollar auf 12,3\%. Für Einkommen über 1 Mio. Dollar steigt der Satz von 10,3\% auf 13,3\%. Die zusätzlichen Einnahmen von ca. 6 Mrd. Dollar jährlich sind für die Schulen vorgesehen. ${ }^{32}$ Die Initiative wurde insbesondere vom demokratischen Gouverneur Jerry Brown, der League of Women Voters, der demokratischen Partei und den Gewerkschaften favorisiert. Gegner waren u.a. Steuerzahlervereinigungen, Unternehmerverbände und die republikanische Partei. ${ }^{33}$ Im Abstimmungskampf kamen enorme Beträge zum Einsatz. Die Befürworter mobilisierten ca. 91 Mio. Dollar, die Gegner 104 Mio. ${ }^{34}$ Die Vorlage gewann mit 55,4\%.

\section{Vgl. o. B.I.}

30 Vgl. detailliert zu Entstehung und Kampagne dieser Vorlage D. DiSarro/W. Hussey, California's 2012-2013 Budget: Brown's Budget Gamble, in: Journal of California Politics and Policy 5 (2013), S. $361 \mathrm{ff}$.

31 Tax Foundation, State and Local Stales Tax Rates, 2011-2013, 14.3.2013, http:// taxfoun-dation.org/article/state-and-local-sales-tax-rates-2011-2013 (6.7.2014).

32 Bowen, Guide 2012 (Fn. 26), S. 12 ff., 80 ff.

33 Vgl. näher Ballotpedia, California Proposition 30, Sales and Income Tax Increase (2012), http://ballotpedia.org/California_Proposition_30,_Sales_and_Income_ Tax _ Increase \%282012\%29 (10.4.2013).

34 National Institute on Money in State Politics, Follow the Money, http://beta.followthemo-ney.org/entity-details?eid=13690362 (24.6.2014) 


\section{Höhere Einkommensteuer für „Multistate Businesses“}

Eine weitere, vom Volk 2012 angenommene Steuererhöhung sieht Proposition 39 vor. Diese Gesetzesinitiative erreichte 61\% Zustimmung. Sie schreibt vor, dass das zu versteuernde Einkommen eines Unternehmens, das in mehreren Gliedstaaten aktiv ist, auf der Basis des in Kalifornien erzielten Umsatzes zu berechnen ist. Dies führt zu jährlichen Mehreinnahmen von einer Mrd. Dollar. Diese sind für Energieeffizienz und das Schulsystem zu verwenden. ${ }^{35}$ Die Befürworter gaben für den Abstimmungskampf mehr als 31,5 Mio. Dollar aus, die Gegner lediglich 90.000. Allein der Milliardär Thomas Steyer steuerte 29,5 Mio. Dollar bei. ${ }^{36}$ Ursprünglich gegnerische Konzerne gaben ihren aktiven Widerstand auf. ${ }^{37}$

\section{Ausgabensenkung}

Der Anteil des Staatshaushalts, der für den Strafvollzug ausgegeben wird, ist in den letzten 40 Jahren horrend gestiegen. Er betrug 2011 10,5\%. Dies ist eine eklatante Fehlallokation staatlicher Mittel. Einer der Hauptgründe liegt in der Gesetzesinitiative Proposition 184 von 1994. Dieses „Three Strikes and You're Out Law" erhöhte die obligatorischen Strafen für Rückfalltäter. Diese machten 2010 26,4 \% aller Strafgefangenen aus. ${ }^{38} 2012$ war die Gesetzesinitiative Proposition 36 mit 69,3\% Ja-Stimmen erfolgreich. Sie führte das Three Strikes-Law stark zurück. Dies ergibt Einsparungen von ca. 70 bis 90 Mio. Dollar jährlich. ${ }^{39}$ Initiatoren waren u.a. Personen, die in der Strafverfolgung tätig sind. Gegner war u.a. die republikanische Partei. ${ }^{40}$

35 Bowen, Guide 2012 (Fn. 26), S. 68 ff., 125 ff.; Ballotpedia, California Proposition 39, Income Tax Increase for Multistate Businesses (2012), http://ballotpedia.org/ California_Proposition_39,_Income_Tax_Increase_for_Multistate_Businesses $\% 282012 \% 29$ (10.4.2013).

36 National Institute on Money in State Politics (Fn. 34), http://beta.followthemoney. org/ entity-details?eid=13690360 (24.6.2014).

37 Ballotpedia, California Proposition 39 (Fn. 35).

38 Heußner, Krise (Fn. 1), S. 203 ff.

39 Bowen, Guide 2012 (Fn. 26), S. 48 ff., 105 ff.

40 California Proposition 36, Changes in the „Three Strikes“ Law (2012), http://ballotpedia.org/California_Proposition_36,_Changes_in_the_\%22Three_Strikes\%22_L aw_\%282012\%29(10.4.2013). 


\section{Wirkungen der Gesetzesänderungen und Fazit für Deutschland}

Die dargestellten Reformen haben dazu beigetragen, dass Kalifornien seit 2010 „die Kurve bekommt“ und ein Ende der kalifornischen Krise absehbar ist. So haben die Reformen des Wahlrechts geholfen, dass Kalifornien 2012 das kompetitivste Wahlsystem aller US-Staaten hatte. ${ }^{41}$ Sodann wurden die Staatshaushalte seit 2011, nachdem die Hürde einer notwendigen Zweidrittelmehrheit im Parlament 2010 abgeschafft worden war, jedes Jahr rechtzeitig verabschiedet. ${ }^{42}$ Dies ist ein Grund, warum das Vertrauen in die Arbeit der kalifornischen Legislative seit dem Tiefpunkt 2010, als lediglich 9\% der Bürger mit der Arbeit des Parlaments zufrieden waren, ${ }^{43}$ kontinuierlich auf mittlerweile $36 \%$ gestiegen ist. ${ }^{44}$ Mit diesem Vertrauenszuwachs einher ging bei den Abstimmungen und Wahlen 2012, dass zum einen die Wähler temporären Steuererhöhungen zustimmten, die zum Ausgleich des Haushalts und

41 D.B. Wood, California a political model? Golden State has most competitive elections, in: Christian Science Monitor v. 12.9.2012, http://www.csmonitor.com/ USA/Politics/2012/0912/California-a-political-model-Golden-State-has-most-competitive-elections (12.4. 2013). E. McGhee/D. Krimm, California's new electoral reforms: the fall election, 8.11.2012, unter: http://www.ppic.org/content/pubs/jtf/ JTF_1112ElectoralReformsJTF.pdf (2.7. 2014). Zu der von der Bürgerkommission in Kalifornien vorgenommenen Wahlkreisneueinteilung 2011 vgl. V. Kogan/E. $M c G h e e$, Redestricting California: An Evaluation oft the Citizens Commission Final Plans, in: California Journal of Politics and Policy 4 (2012), S. 1 ff. Für die Wahlen 2014 liegt der „Competitiveness Index“ noch nicht vor, http://ballotpedia.org/A_ $\% 22$ Competitiveness_Index $\% 22$ _for_capturing_competitiveness_in_state_legislative_elections (23.6.2014).

42 J.E. Gould, California Miracle: An On-Time Budget!, in: Time v. 30.6.2011, http://con-tent.time.com/time/nation/article/0,8599,2080637,00.html (22.3.2013); C. Megerian, Gov. Jerry Brown signs California Budget, in: Los Angeles Times v. 27.6.2012, http://latimesblogs.latimes.com/california-politics/2012/06/governorjerry-brown-signs-california-budget.html (25.6.2014); W. Buchanan, Gov. Brown proudly signs balanced state budget, in: San Francisco Chronicle v. 27.6.2013, http://www.sfgate.com/news/article/Gov-Brown-proudly-signs-balanced-state-budget-4628307.php\#item-20204 (25.6.2014); D. Siders, Gov. Brown signs on-time budget, giving his re-election campaign a boost, in: The San Francisco Bee v 20.6. 2014, http://www.sacbee.com/2014/06/20/6500384/gov-brown-signs-on-time-budget.html (25.6.2014).

43 Vgl. Heußner, Krise (Fn. 1), S. 225.

44 Bezogen auf die Bürger, die wahrscheinliche Wähler sind, vgl. M. Baldassare/D. Bonner/ S. Petek/J. Shrestha, Californians \& their government, May 2014, S. 7, http://www.ppic. org/content/pubs/survey/S_514MBS.pdf (25.6.2014). Das Vertrauen in den US-Kongress beträgt nur 14\%, dies., ebda., S. 17. 
zugunsten des Bildungssystems notwendig waren. ${ }^{45}$ Zum anderen gewannen die Demokraten in beiden Häusern des Parlaments eine Zweidrittelmehrheit. ${ }^{46}$ Solche Mehrheiten gab es zuletzt $1933 .{ }^{47}$ Sie konnten bisher von den Republikanern gehaltene Sitze u.a. deshalb dazu gewinnen, weil die Demokraten die Haushalte 2011 und 2012 allein verabschiedet hatten ${ }^{48}$ und so allein in den Genuss des Vertrauenszuwachses kamen. „It puts Republicans out in the cold. “49 Die Minderheit konnte seit 2011 nicht mehr auf der Bremse stehen.

Es mag fraglich sein, welchen Beitrag die durch direkte Demokratie herbeigeführten kalifornischen Wahlreformen der letzten Jahre zur Verbesserung der kalifornischen politischen Performance beigetragen haben. ${ }^{50}$ Es könnte sein, dass diese Reformen erst mit der Zeit greifen. ${ }^{51}$ Unzweifelhaft hat Volksgesetzgebung jedoch zu höheren Steuern und geringeren Ausgaben und zu einer vernünftigen Lockerung der Term Limits geführt. Dies dürfte es in den kommenden Jahren einfacher machen, den kalifornischen Haushalt auszugleichen. ${ }^{52}$ Nach den derzeitigen Prognosen wird der Haushalt stetig

45 Vgl. Megerian, Brown (Fn. 42).

46 Brownstein (Fn. 4).

47 Und zwar für die Republikaner, s. Glossary of legislative terms, Appendix M, California Legislature Partisan Composition, http://www.leginfo.ca.gov/pdf/2011/2011 Glossary_Appendices_Index.pdf (2.7.2014).

48 C. Megerian, California Legislature passes \$92.1-billion budget, in: Los Angeles Times v. 16.6.2012, http://articles.latimes.com/2012/jun/16/local/la-me-state-budget -20120616 (25.6.2014).

49 H. Brady, Dekan der Goldman School of Public Policy, University of California, Berkeley, zit. in: Gould, Miracle (Fn. 42).

50 Vgl. E. McGhee/M. Baldassare, Is California Turning a Corner?, Jan. 2014, S. 1, 3, http://www.ppic.org/content/pubs/report/R_114EM2R.pdf (25.6.2014); The Economist v. 27.1.2014, California political reforms. Rewriting the rules, http://www.economist.com/blogs/democracyinamerica/2014/01/californias-political-reforms (23.6. 2014); A. Nagourney, California Sees Gridlock Ease in Governing, in: New York Times v. 18.10.2013, http://www.nytimes.com/2013/10/19/us/california-upends-itsimage-of-legislative-dys-function.html?pagewanted=all\&_r=0 (23.6.2014); McGhee et al. (Fn. 5), S. 337 ff.; T. Kousser/J.H. Phillips/B. Shor, Reform and Representation: Assessing California's Top-Two Primary and Redistricting Commission, 2013, S. 1 ff., http://www.columbia.edu/ jhp2121/workingpapers/ReformAndRepresentation.pdf (4.7.2014); D. Schnur, A Rebuttal - Redistricting Reform and Top-Two Primary, in: Fox\&Hounds v. 16.8.2012, http://www.foxandhoundsdaily.com/2012/ 08/a-rebuttal-redistricting-reform-and-top-two-primary/\#sthash.Fgx5gSLW.dpuf (4.7.2014).

51 Kousser u.a., Reform (Fn. 50), S. 25.

52 DiSarro/Hussey, Budget (Fn. 30), S. 397. Vgl. auch M. Koch, Kalifornien gegen Texas, in: SZ v. 9./10.2.2013, S. 23. 
Überschüsse produzieren. ${ }^{53}$ Außerdem ist die durch Volksgesetzgebung zustande gekommene Abschaffung der notwendigen Zweidrittelmehrheit für Haushaltsbeschlüsse epochal. Insofern steht fest, dass Volksgesetzgebung einen starken Beitrag geleistet hat, die kalifornische Krise zu überwinden.

Für die direktdemokratische Diskussion in Deutschland bedeutet die aktuelle kalifornische Entwicklung ein weiteres Mal, dass „das Beispiel Kalifornien“" nicht als wohlfeiles Argument gegen die Einführung von Volksgesetzgebung auf Bundesebene taugt. Denn trug schon zur Entstehung der „kalifornischen Krise" nicht Volksgesetzgebung an sich bei, sondern die spezielle kalifornische Ausprägung dieser Gesetzgebungsart, ${ }^{54}$ so zeigt die aktuelle Entwicklung, dass sich selbst unter den suboptimalen Bedingungen von Volksgesetzgebung „California style“ direktdemokratische Selbstheilungskräfte entfalten können.

53 Unterstellt, dass bestimmte Annahmen eintreten, näher M. Taylor, Legislative Analyst, California's Fiscal Outlook, Nov. 2013, S. 3-6, http://www.lao.ca.gov/ reports/2013/bud/fiscal-outlook/fiscal-outlook-112013.pdf (3.7.2014).

54 Ausführlich Heußner, Krise (Fn. 1), S. 176 ff., 231. 
